\title{
A rare case of oncocytoma of the parotid gland with aggresssive features and malignant potential: a case report
}

\begin{abstract}
We report a rare case of an oncocytoma of the parotid gland with borderline histological features meaning with possible agressive behavior. A 45 year old male presented to the Otolaryngology department with a unilateral small swelling in the right parotid region. $\mathrm{CT}$ and MRI studies revealed a right parotid mass and moderate enhancement following contrast media injection. The patient was treated with superficial parotidectomy with complete tumor resection. Postoperative course was uneventful. Histological results revealed a rare case of an oncocytoma with possible malignant potential. Close clinical and radiological follow-up in this case is of paramount importance. This case is unique due to the few existing references in literature.
\end{abstract}

Keywords: oncocytoma, parotid gland, borderline features, malignant potential
Volume 10 Issue 2 - 2018

\author{
Nikolopoulos Elias,' Ploumidou Katherine,' \\ Gazalidou Maria, ${ }^{2}$ Giannakou Niki ${ }^{2}$ \\ 'Department of Otolaryngology Athens Medical Center \\ (Omilos latrikou Athinon) Greece \\ ${ }^{2}$ Department of Pathology Athens Medical Center (Omilos \\ latrikou Athinon) Greece
}

Correspondence: Katherine Ploumidou, Department of Otolaryngology Head and Neck Surgery Athens Medical Center (AMC), Distomou II Amarousio I5I-25 Athens Greece, Greece, Tel +306932722273, Email catplou@yahoo.gr

Received: March II, 2018 | Published: April 23, 2018

\section{Introduction}

Oncocytic neoplasms belong to a group of infrequent tumors of the salivary glands just about $0,5 \%-1,5 \%$ and according to WHO they are histologically categorized in three distinct types, to be specific oncocytosis, oncocytoma and oncocytic carcinoma. ${ }^{1}$

Oncocytomas are scarce benign epithelial tumors that most commonly occur in adults between the sixth through the eighth decades of life. They are painless tumors and distribution between genders is equal. ${ }^{2}$ Majority of tumors occurs in the parotid gland and in addition it is the most common salivary gland that contains these neoplasms at a percentage of $78-80 \%$ in its superficial lobe. Oncocytes may be found individually or in groups in minor salivary glands. They are also found in the pancreas, in the respiratory tract, in thyroid, in parathyroid, pituitary and adrenal glands. Those originating from minor salivary glands have less well defined borders and are not encapsulated. ${ }^{2}$ Alternative designations for the tumor are oncocytic adenoma, oxyphilic granular-cell adenoma and oxyphilic adenoma. ${ }^{3}$

An oncocytoma is characterized pathologically as a well defined delineated encapsulated mass constituted of layers of oncocytes (small round nucleus, microgranullar eosiniphilic cytoplasm rich in mitochondria). ${ }^{5}$ The surface is usually pink to rust in color. The tumor usually has a granulated appearance due to the abundant and hyperplastic mitochondria, a characteristic which cannot be observed in other tumors. An oncocytoma does not contain any lymphoid tissue. $^{2}$

The clinical traits of oncocytomas bear resemblance to those of other benign and low grade salivary gland tumors making clinical diagnosis very difficult and demanding. Patients characteristically present a solitary slow growing painless parotid mass and manifest usually on MRI imaging T1 and T2 hypointensity with homogeneous contrast enhancement. ${ }^{3}$ The recommended treatment of choice is thorough surgical excision either with superficial or total parotidectomy. The range and dimension of excision is imposed by preoperative clinical, radiological examinations (CT and MRI) and intraoperative findings. In addition a follow-up MRI at 12 and 24 months is usually suggested in order to evaluate patient progression. Radiation therapy is not recomended because oncocytes are radioresistant. ${ }^{3-5}$

\section{Case report}

A 45 year old male was presented at the Otolaryngological Department with a small swelling in the right parotid region. No pain or signs of infection were present. Clinical examination revealed a well defined soft mass with no palpable cervical lymph nodes. CT and MRI showed a well circumscribed mass with a major probability of a benign tumor in the superficial lobe of the right parotid gland. The patient underwent superficial parotidectomy with complete tumor excision and preservation of the facial nerve under general anaesthesia.

Pathological examination of the tissue sections revealed a $1,5 \mathrm{~cm}$ circumscribed neoplasm with tan color partially encapsulated, covered by healthy parotid tissue. A lymphe node $2,1 \mathrm{~cm}$ was also present but had non specific reactive changes. The neoplasm had a nested pattern of growth suggesting an oncocytoma. It composed of cells with vesicular nuclei, with prominent nucleoli and abundant granular acidophilic cytoplasm. (Figure 1) (Figure 2). Enhanced mitosis, cytological atypia, moderate nuclear pleomorphism and necrosis were noted. Focally an abnormal circumference with connective tissue and fibrous elements was also present suggesting that this neoplasm has agressive histological features with malignant potential. Immunohistological staining pattern showed that the neoplastic cells had a Ki67 positive at 8\% (Figure 3), positve for ker 8/18 (Figure 4), positive-negative for $\mathrm{p} 63$ while were negative for ker 14, SMA, CEA, TTF-1, CD31, calcitonin, EMA, GFAP, and S100 staining. 
This is not a typical case of an oncocytoma but an oncocytic neoplasm of uncertain malignant potential. The diagnosis of an oncocytic carcinoma was excluded due to unsufficient presence of major diagnostic criteria for malignancy.

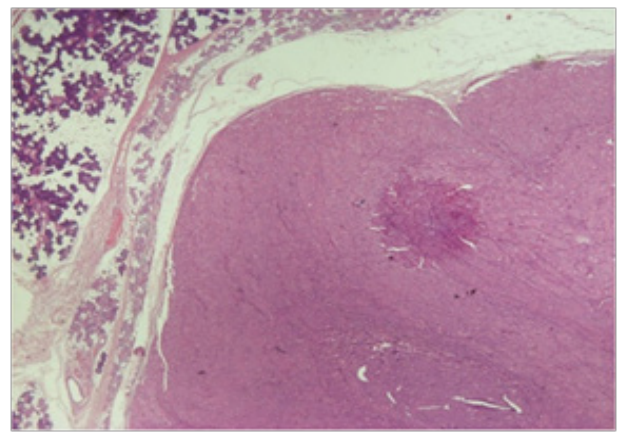

Figure I Ex 2.5:A circumscribed neoplasm covered by healthy tissue.

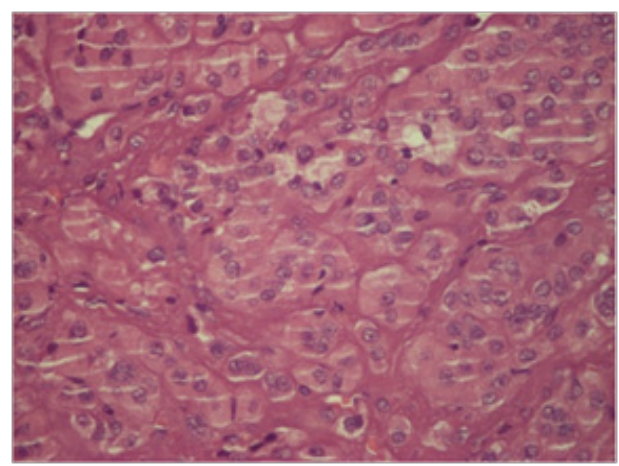

Figure 2 HEx 40: Nested pattern of neoplasm composed of cells with vesicular nuclei and abundant granular acidophilic cytoplasm. Vesicular nuclei with prominent nucleoli.

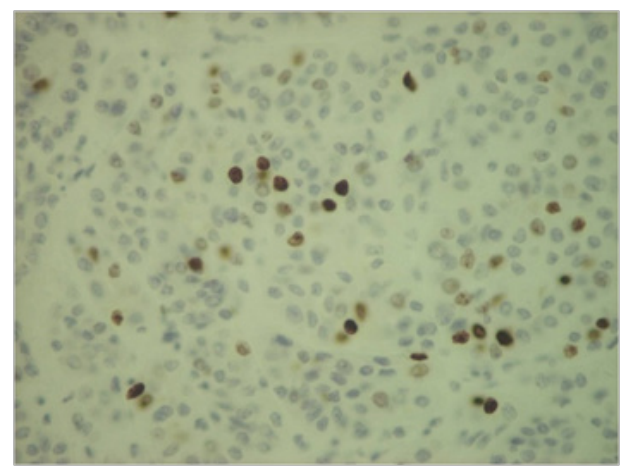

Figure 3 Ki67: Positive at $8 \%$ of neoplastic cells.

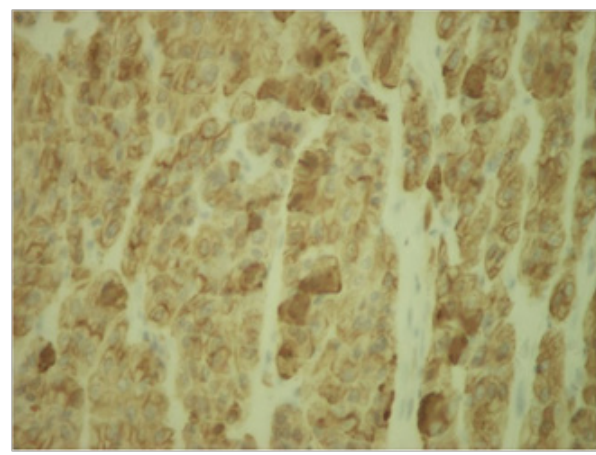

Figure 4 Ker 8//8: Postive at the neoplastic cells.

\section{Discussion}

Oncocytomas chiefly affect the parotid salivary gland. They are usually limited and delineated with a size of over $3 \mathrm{~cm} .{ }^{6}$ Their growth rate is typically slow. Although malignant transformation and local recurrence of oncytomas is unusual and uncommon, clinical followup is very important. ${ }^{5}$ They are composed of layers of oncocytes, epitehlial cells which exhibit specific cytological characteristics namely a central pycnotic nucleus and a granular abundant eosinophlic cytoplasm filled with numerous mitochondria of various sizes. Diagnosis by fine needle aspiration cytology (FNAC) may be very difficult and sensitivity in detection of oncocytic neoplasms is reported to be only $29 \% .{ }^{5,7,8}$ Even though FNAC has been used as a primary screening tool for salivary gland lesions with high levels of sensitivity and specificity, salivary glands do have the tendancy for having overlapping morphological features, therefore differentiating oncocytoma from oncocytic carcinoma can be difficult on cytology as the former can appear cytologically malignant looking, while the latter can look deceptively benign. Furthermore, differentiating oncocytosis from oncocytoma in cytology is difficult not to mention unattainable. Histopathology still remains the gold standard to presume the precise diagnosis. ${ }^{7}$ Specifically the nature of the tumor cells is usually confirmed with special methods, such as histochemical or immunochemical stains. Presence of pleomorphism, necrosis, perineural spread, frequent mitosis, intravascular invasion and distant metastasis to cervical nodes, kidneys, lungs and mediastinum as well as extensive invasion and destruction of adjacent structures are major signs of a high grade malignant tumor. ${ }^{9}$

According to the WHO two criteria are necessary in order to clinch the diagnosis of oncocytic carcinoma. The first consists of the pathological recognition of tumor cells as oncocytes and the second consists of the pathological finding of local infiltration and metastasis and presence of connective tissue. Oncocytic carcinoma shows a greater mitotic reactivity, activity and more nuclear pleomorphism compaired to a benign oncocytoma. ${ }^{10}$ It has also been reported that using an antmitochondrial antibody when performing immunohistochemistry can identify with high sensitivity and specificity the mitochondria on light microscopy. Oncocytic carcinomas pathologicaly display immunoreactivity for mitochondria and are characterised by atypical accumulation of these organelles. This atypical accumulation is a compensatory mechanism to intrinsic functional defects of the mitochondria. The defective mitochondria produce high amounts of reactive oxygen species which predispose to genome instability and cellular transformation. Management and treatment of oncocytic carcinomas is still elusive and not well established due to the low incidence of this tumor type. Current treatment consists of surgical intervention with facial nerve sacrifice followed by immediate grafting and radiotherapy as well as prophylactic neck dissection. Eficacy of radiotehrapy still remains unclear., ${ }^{9,11}$

In this specific case while the tumor exhibits cytologic atypia, abnormal circumference and increased mitotic activity, atypical mitotic and invasive features were not identified. Even though the major diagnostic criteria for malignancy were not satisfied, the presence of moderate nuclear pleomorphism and an abnormal circumference, indicate that the neoplasm is compatible with a tumor of borderline (uncertain) malignant potential. Due to the nature of this neoplasm, close clinical and radiological follow-up of the patient is of paramount importance. This is probably one of a few cases of 
oncocytoma with borderline features and malignant potential that has been reported in literature.

\section{Conclusion}

In summary we present a rare case of oncocytoma with aggressive features and malignant potential. Oncocytomas should be evaluated using CT and MRI studies in order to assess the extent of their presence but the final diagnosis should be confirmed histopathologicaly and by immunostaining. Further close follow-up of the patient is recommended and is of paramount importance.

\section{Acknowledgements}

None.

\section{Conflict of interest}

The authors have no funding, financial relationships or conflicts of interest to disclose.

\section{References}

1. Seifert G. Tumor like lesions of the Salivary Glands.The New WHO classification. Pathol Res Pract. 1992;88(7):836-846.

2. Calzada GG, Hanna YE. Benign Neoplasms of Salivary Glands. In: Cummings Otolaryngology Head and Neck Surgery 5th ed. 2010. 1171 p.

3. TJ Tan, TY Tan. CT Features of Parotid Gland Oncocytomas. A Study of 10 cases and Literature review. AJNR Am J Neuroradiol. 2010;31(8):1413-1417.
4. Sepulveda I, Platin E, Spencer ML, et al. Oncocytoma of the Parotid Gland. A case report and review of literature. Case Rep Oncol. 2014;7(1):109-116.

5. Popovski V, Benedetti A, Monevska DP, et al. Oncocytoma of the Deep Lobe of the Parotid Gland. Open Access Maced J Med Sci. 2016;4(2):290-292.

6. Evren C, Demirbilek N, Yigit VB, et al. Oncocytoma of the Parotid Gland complicated by Hypercalcemia : a case report. Kulak Burun Bogaz Intis Derg. 2015;25(3):179-181.

7. Chakrabart I, Basu A, Ghosh N. Oncocytic lesion of Parotid gland: a dilemma for cytopathologists. J Cytol. 2012;29(1):80-82.

8. Liao X, Haghihi P, Coffey C, et al. A Rare Case of Exclusive Oncocytic Mucoepidermoid Carcinoma with MAML2 Translocation. Rare Tumors. 2016;8(2):6166.

9. Oghan F, Apahan T, Guvey A. Rare Malignant Tumors of the Parotid Glands: oncocytic neoplasms. In: Neck Dissection-Clinical Applications and Recent Advances. 2012. p. 137-148.

10. Sciubba JJ, Shimono M. Oncocytic Carcinoma. In: Barnes L, editors. WHO Classification of Tumors Pathology and Genetics Head and Neck Tumors Lyon: IARC Press; 2005. 235 p.

11. Elis GL, Auclair PL. Tumors of Salivary Glands. In: Atlas of Tumor Pathology 3rd series fasiscle 17. Washignton DC: Armed forces Institute of Pathology; 1996. p. 318-324. 